INPLASY

PROTOCOL

To cite: Cui et al. Incidences of Adverse Reactions in SARS-

CoV-2 mRNA Vaccines: A

Meta-analysis. Inplasy protocol 202210124. doi:

10.37766/inplasy2022.1.0124

Received: 26 January 2022

Published: 26 January 2022

Corresponding author:

HuaiXing Cui

cuihuaixing@163.com

Author Affiliation:

Tianjin University of Traditional Chinese Medicine.

Support: There is no support.

Review Stage at time of this submission: Formal screening of search results against eligibility criteria.

Conflicts of interest:

None declared.

\section{Incidences of Adverse Reactions in SARS-CoV-2 mRNA Vaccines: A Meta-analysis}

Cui, HX1; Zeng, XY2; Zhang, NJ3; Sun, X4; Guan, YY5; Bu, HE6

Review question / Objective: This study searched PubMed, Web of Science, Embase to collect healthy people aged 12 and older, subjects with no previous history of COVID-19 infection, $A$ randomized controlled trial of mRNA vaccine versus placebo. Using RevMan5.4 software, meta-analysis was conducted to compare the effects of injection of mRNA vaccine and placebo on the incidence of adverse reactions in healthy over 12 years of age. Main indexes include the incidence of local adverse reactions (including injection site swelling), the incidence of systemic adverse reactions, (including fever, fatigue, chill). So as to provide a reference for clinical practice.

Information sources: Literature published before December 1, 2021 in Pub Med, Embase, Web of Science.

INPLASY registration number: This protocol was registered with the International Platform of Registered Systematic Review and Meta-Analysis Protocols (INPLASY) on 26 January 2022 and was last updated on 26 January 2022 (registration number INPLASY202210124).

\section{INTRODUCTION}

Review question / Objective: This study searched PubMed, Web of Science, Embase to collect healthy people aged 12 and older, subjects with no previous history of COVID-19 infection, A randomized controlled trial of mRNA vaccine versus placebo. Using RevMan5.4 software, metaanalysis was conducted to compare the effects of injection of mRNA vaccine and placebo on the incidence of adverse reactions in healthy over 12 years of age. Main indexes include the incidence of local adverse reactions (including injection site swelling), the incidence of systemic 
adverse reactions,(including fever, fatigue, chill). So as to provide a reference for clinical practice.

Condition being studied: COVID-19, caused by severe acute respiratory syndrome Type 2 coronavirus (SARS-COV-2), was first reported in China on 31 December 2019. This is the most extensive global pandemic to hit humanity in nearly a century, posing a serious crisis and a severe test for the world. Human life, safety, and health are seriously threatened. Vaccines are considered to be the most effective means of controlling Novel coronavirus infection due to the lack of effective antiviral drugs and the persistence of root control of the outbreak. At present,countries around the world are urgently developing COVID-19 vaccines, mainly including the following six technical routes: inactivated vaccine, attenuated live vaccine, protein subunit vaccine, viral vector vaccine (replication/ non-replication), nucleic acid vaccine (DNA, mRNA), and virus particle like a vaccine. Among them, due to its excellent characteristics of mRNA vaccine and high effectiveness in clinical trials, it is widely used in Europe and The United States. Currently, the safety and immunogenicity of mRNA vaccines in phase I / II, II and II / III clinical trials have been published, showing efficacy. However, the safety of BNT162b2 remains controversial. Therefore, this study systematically evaluated the safety of mRNA vaccines, in order to provide a reference for its clinical application.

\section{METHODS}

Search strategy: We searched for literature published before December 1, 2021 in Pub Med, Embase, Web of Science, using the following search terms "(effectiveness OR safety) AND (sars-cov-2 vaccine OR 2019ncov vaccine OR covid-19 vaccine)"in WOS and PubMed, "(effectiveness:ab,ti OR safety:ab,ti) AND ('sars-cov-2 vaccine':ti OR '2019-ncov vaccine':ti OR 'covid-19 vaccine':ti) AND [clinical study]/lim" in Embase.
Participant or population: Healthy adults aged $>12$ years with no previous history of COVID-19 infection.

Intervention: The intervention of the experimental group was mRNA vaccine.

Comparator: The intervention in the control group was a placebo injection.

Study designs to be included: The study type was a randomized controlled study(RCTs).

Eligibility criteria: The PICOS (population, intervention, comparator, outcome and study) design approach was usedwas used to define study eligibility criteria. Population--Subjects enrolled in a clinical trial related to SARS-CoV-2 vaccine. Intervention - -SARS-CoV-2 vaccination. Comparator--SARS-CoV-2 placebo. Outcomes--Vaccine safety for SARS-CoV-2 was assessed based on the incidence of adverse reactions. The results were the safety of the vaccine, including adverse events at the injection site (pain.swelling), systemic adverse events (e.g. fever, fatigue, chills) and serious adverse events (e.g. dehydration, syncope, atrial fibrillation, pulmonary embolism, acute kidney injury, etc.).Subjects were asked to record any adverse events at days 0 to 7,14 or 28 after injection. Study design - - randomized controlled trials were eligible for inclusion. Animal trials, case reports, reviews, editorials, letters and meeting abstracts were excluded.

Information sources: Literature published before December 1, 2021 in Pub Med, Embase, Web of Science.

Main outcome(s): The incidence of local adverse reactions (including redness, swelling); Incidence of systemic adverse reactions (including fever, fatigue, cough).

Data management: We use Endnote $X 9$ to manage papers.

Quality assessment / Risk of bias analysis: Two researchers will assess study quality 
across seven domains using the Cochrane Risk of Bias Tool. If any different points of view emerge, another experienced researcher will help resolve them through discussion.

Strategy of data synthesis: RevMan $\mathbf{5 . 4}$ software will be used for data analysis. The dichotomous data will be expressed as risk ratio (RR) and $95 \%$ confidence interval $(\mathrm{Cl})$, and the continuous data will be estimated as mean difference (MD) or standard mean difference (SMD) and $95 \% \mathrm{Cl}$. Statistical heterogeneity of conformity tests was examined by the $I^{2}$ test. If in enough eligible studies there is little heterogeneity $\left(I^{2} \leq 50 \%\right)$ of the same results, the data are aggregated using a fixed-effects model, and meta-analysis is performed. If there is significant statistical heterogeneity between the included studies $\left(I^{2}>50 \%\right)$, the random-effects model isused to summarize the data and subgroup analysis is used to determine its source. If we cannot examine the source of apparent heterogeneity, we will use descriptive analysis rather than meta-analysis. The results of the metaanalysis will be displayed through the forest map Sensitivity and subgroup analyses were performed to explore whether significant heterogeneity existed. Two researchers will assess study quality across seven domains using the Cochrane Risk of Bias Tool. If any different points of view emerge, another experienced researcher will help resolve them through discussion.

Subgroup analysis: We will perform subgroup analysis according to the different details of interventions, study quality and outcome indicators.

Sensitivity analysis: Sensitivity analysis: We will perform sensitivity analysis based on sample size, research design, heterogeneity quality, methodological quality and statistical model, excluding trials with low quality, and ensure the stability of analysis results.

Language: Only English literature was included.
Country(ies) involved: China.

Keywords: SARS-CoV-2 vaccines, safety, meta-analysis, Adverse events.

Dissemination plans: We plan to publish this meta-analysis in relevant scientific journals and disseminate it widely on the Internet.

Contributions of each author:

Author 1 - HuaiXing Cui - Drafted the manuscript; Methodology; Software.

Email: cuihuaixing@163.com

Author 2 - YueXlian Zeng - Data selection; Data extraction.

Author 3 - NaiJin Zhang - Quality assessment.

Author 4 - Xuan Sun - Quality assessment. Author 5 - YuanYuan Guan - Quality assessment.

Author 6 - HuaiEn Bu - The author read, provided feedback and approved the final manuscript.

Email: huaienbu@tjutcm.edu.cn 